\title{
Meningitis por Streptococcus pneumoniae en paciente pediátrico previamente inmunizado: reporte de un caso
}

\section{Streptococcus pneumoniae meningitis in a previously immunized pediatric patient: report of a case}

Correspondencia Ingrid Tovar Ibarra intoib@hotmail.com

\section{Recibido: 27/04/2017}

Arbitrado por pares

Aprobado: 26/07/2017

Citar como: Tovar Ibarra I, Romero Guzmán A. Meningitis por Streptococcus pneumoniae en paciente pediátrico previamente inmunizado: reporte de un caso. Acta Med Peru. 2017;34(3):217-20

\section{Ingrid Tovar Ibarra ${ }^{1}$, Alberto Romero Guzmán ${ }^{1}$}

1 Servicio de Medicina B, Instituto Nacional de Salud del Niño. Lima, Perú.

\section{RESUMEN}

La meningitis bacteriana constituye una importante causa de morbimortalidad en el mundo y, a pesar de que la incidencia se ha reducido en los años posteriores a la inclusión de la vacuna antineumocócica en los calendarios de inmunizaciones, no se ha podido evitar el surgimiento de cepas emergentes. Presentamos el caso de una paciente de 5 años con inmunizaciones completas para la edad, con un cuadro clínico sugerente de meningoencefalitis, cuya evolución (con gran compromiso sistémico y pobre respuesta al tratamiento) nos hizo sospechar de la presencia de cepas emergentes y/o resistencia antimicrobiana, en el planteamiento de interrogantes respecto a estados de inmunodeficiencia o respuesta inadecuada a la vacunación, el impacto que realmente tiene la vacuna en nuestro país y la necesidad de realizar una más estricta vigilancia epidemiológica para la identificación de serotipos emergentes.

Palabras clave: Vacunas neumococicas; Resistencia microbiana a antibióticos; Programas de inmunización; Meningitis (fuente: DeCS BIREME).

\section{ABSTRACT}

Bacterial meningitis is an important cause of morbidity and mortality all over the world; and, in spite of a reduction in its incidence because of the introduction of an anti-pneumococcal vaccine in the immunization programs in many places, the occurrence of emerging strains has not been stopped. We present the case of a 5-year old girl with complete immunizations for her age, with a clinical condition suggesting meningoencephalitis, whose progress and outcome (significant systemic involvement and poor response to therapy) lead us to think in the possibility of emerging strains and/or resistance to antibacterial agents, and also to ask ourselves about an underlying immune deficiency or inadequate response to vaccination, the real impact of the vaccine in our country, and the need for performing more stringent epidemiological vigilance in order to identify emerging serotypes.

Keywords: Pneumococcal vaccines; Drug resistance, microbial; Immunization programs; Meningitis (source: MeSH NLM). 


\section{INTRODUCCIÓN}

La meningitis es una enfermedad grave, considerada una emergencia médica. Ante su sospecha, es indispensable iniciar inmediatamente terapia antimicrobiana $y$, a la brevedad, determinar el agente causal y la sensibilidad al medicamento elegido. La mortalidad de la meningitis no tratada se acerca al $100 \%$; incluso con la terapia óptima, las secuelas neurológicas son frecuentes ${ }^{[1]}$.

Streptococcus pneumoniae es el agente etiológico bacteriano más frecuente en lactantes mayores de 1 mes y niños, a pesar de la significativa disminución de su incidencia posterior a su inclusión en el calendario de vacunación ${ }^{[2]}$. Así mismo, el surgimiento de cepas de Streptococcus pneumoniae resistente a penicilina conlleva a una menor sensibilidad a cefalosporinas, haciendo más difícil el tratamiento de las infecciones neumocócicas, especialmente las del sistema nervioso central ${ }^{[3]}$.

Se presenta el caso clínico de una paciente preescolar de 5 años, previamente sana, sin déficit inmunológico y con vacunación antineumocócica completa, con meningitis causada por Streptococcus pneumoniae; además, se discute respecto a las características clínico epidemiológicas de este agente, haciendo énfasis en la necesidad de tomar medidas de salud pública para la reducción de la prevalencia de esta enfermedad inmunoprevenible.

\section{REPORTE DE CASO}

Paciente mujer de 5 años de edad, con antecedentes de cirugía de drenaje de hematoma epidural parietal bilateral por traumatismo craneoencefálico ( 6 meses antes del evento actual, por ello se consideró la realización de tomografía cerebral en busca de abscesos y/o hematomas), desarrollo psicomotor normal e inmunizaciones completas para la edad, incluyendo las 3 dosis de vacuna antineumocócica del tipo heptavalente. No obstante, también debemos considerar que no toda vacuna es $100 \%$ eficaz y que factores externos podrían afectar su adecuada acción (alteraciones en la preservación de la vacuna, etc.).

Cursó con un tiempo de enfermedad de 2 días, caracterizado por fiebre, vómitos y tendencia al sueño; fue diagnosticada de faringoamigdalitis un día antes del ingreso, iniciándose tratamiento con amoxicilina. Al persistir sintomatología y asociarse inestabilidad para la marcha, acudió a emergencia y fue hospitalizada.

A la exploración, la paciente tenía un peso de $21,8 \mathrm{~kg}$, talla de $118 \mathrm{~cm}$, frecuencia cardiaca de 100 latidos por minuto, frecuencia respiratoria de 30 por minuto, temperatura de $36,5^{\circ} \mathrm{C}$, saturación de $\mathrm{O}_{2}$ de $100 \%$ a $\mathrm{FiO}_{2}$ ambiental. La orofaringe estaba congestiva con presencia de exudado. La paciente se encontraba dormida, poco reactiva a estímulos. Glasgow $12 / 15$, rigidez de nuca. Resto del examen físico no contributorio.

Al ingreso, los exámenes auxiliares destacaron la presencia de desviación izquierda sin leucocitosis, creatinina incrementada, acidosis metabólica, trastorno de coagulación y proteína C reactiva incrementada. El estudio de líquido cefalorraquídeo mostró proteinorraquia $(345 \mathrm{mg} / \mathrm{dl})$, hipoglucorraquia (0 $\mathrm{mg} / \mathrm{dl}), 45$ leucocitos a predominio de mononucleares; así como presencia de abundantes diplococos grampositivos, coaglutinaciones y cultivos positivos a Streptococcus pneumoniae sensible a cefalosporinas y vancomicina, resistente a penicilinas y macrólidos.

En emergencia se inició tratamiento con ceftriaxona, vancomicina, dexametasona, manitol y fenitoína (a sugerencia de neurología, pese a no encontrarse en el protocolo de manejo y la ausencia de convulsiones; por el antecedente de traumatismo craneoencefálico y posibilidad de presencia de hematomas o lesiones focales); cursó con vómitos en proyectil en 10 oportunidades, persistió febril, con criterios de sepsis, falla multiorgánica (insuficiencia renal aguda, coagulopatía) y acidosis metabólica. Persistió trastorno de sensorio. Se realizó tomografía cerebral sin contraste que evidenció edema cerebral marcado, sin signos de hemorragia ni isquemia.

A los 4 días del ingreso, reconoció su nombre, se encontraba parcialmente relacionada con el entorno, aún febril, con escala de Glasgow 14/15; se inició alimentación enteral. Al sexto día se suspendió manitol y fenitoína, persistió febril, con cefalea holocraneana y presencia de signos meníngeos, por lo que se sospechó de la posibilidad de absceso cerebral y se solicitó tomografía cerebral contrastada. A los 7 días del inicio de tratamiento antibiótico y por persistencia de fiebre y signos meníngeos se decidió rotar de ceftriaxona a meropenem y continuar con vancomicina, con el fin de ampliar la cobertura a gérmenes anaerobios, por la sospecha clínica de absceso cerebral.

Se efectivizó tomografía cerebral contrastada, que mostró imagen hiperdensa rodeada de halo hiperdenso en región frontoparietal izquierda, sugerente de absceso cerebral; se decidió ampliar estudio con resonancia magnética y mantener cobertura con meropenem y vancomicina. El informe de resonancia (obtenido 5 días después) no evidenciaba presencia de absceso, sino infarto focal en globus pallidus izquierdo y signos inflamatorios.

A los 14 días, se realizó punción lumbar de control que mostró disminución de proteinorraquia, glucosa normal y pleocitosis marcada a predominio de polimorfonucleares (1182 leucocitos/ $\mathrm{ml}$ ). Además, controles de laboratorio evidenciaron mejoría de reactantes de fase aguda y disminución de leucocitosis, todo ello asociado a mejoría clínica; pero con persistencia de fiebre. Con los resultados de este nuevo estudio de líquido cefalorraquídeo, se decidió mantener tratamiento con meropenem y reiniciar ceftriaxona en lugar de vancomicina (de acuerdo con el antibiograma que indicaba sensibilidad a cefalosporinas).

A los 21 días de tratamiento antibiótico, con último cultivo de LCR negativo, persistía febril y con cefalea. Además, el dosaje de inmunoglobulinas y citometría de flujo se encontraron dentro de parámetros normales, estudio de tuberculosis negativo. A los 23 días de tratamiento se evidenció caída de 
Tabla 1. Evolución de parámetros de laboratorio.

\begin{tabular}{|c|c|c|c|c|c|c|}
\hline \multirow{2}{*}{ Variable } & \multicolumn{6}{|c|}{ Fecha de toma de prueba* } \\
\hline & $30 / 04$ & 01/05 & $02 / 05$ & 06/05 & $12 / 05$ & $24 / 05$ \\
\hline Urea (mg/dL) & 73 & 125 & -- & -- & -- & 55 \\
\hline Creatinina $(\mathrm{mg} / \mathrm{dL})$ & 1,3 & 1,91 & -- & 0,67 & -- & 0,99 \\
\hline Hemoglobina (g/dL) & 13,7 & 8,2 & 9,0 & -- & 10,3 & 11,1 \\
\hline Plaquetas (por mL) & 15000 & 39000 & 24000 & 173000 & 609000 & 41000 \\
\hline Leucocitos (por mL) & 6120 & -- & 26950 & -- & 15720 & 14900 \\
\hline Abastonados (\%) & 12 & -- & 9 & -- & 0 & 0 \\
\hline PCR (mg/dL) & 38 & -- & -- & -- & 1,8 & 8,25 \\
\hline Proteínas en LCR (mg/dL) & 345 & -- & -- & -- & 230 & 100 \\
\hline Glucosa en LCR (mg/dL) & 0 & -- & -- & -- & 63 & 53 \\
\hline Leucocitos en LCR & 45 & -- & -- & -- & 1182 & 3 \\
\hline Mononucleares (\%) & 60 & -- & -- & -- & 30 & 100 \\
\hline PMN (\%) & 40 & -- & -- & -- & 70 & 0 \\
\hline Tiempo de protrombina (s) & 35,4 & 17,5 & -- & -- & 14,6 & 13,8 \\
\hline TTPA (s) & 91,3 & 49,6 & -- & -- & 29,8 & 32,6 \\
\hline TGO (U/L) & -- & 285 & -- & 31 & -- & -- \\
\hline Cultivo de LCR & -- & $\mathrm{CP}$ & -- & -- & -- & -- \\
\hline
\end{tabular}

LCR: Líquido cefalorraquídeo PCR: Proteína C reactiva PMN: Polimorfonucleares TTPA: Tiempo parcial de tromboplastina activada TGO: Transaminasa glutámico oxalacética. CP: Cultivo positivo para Streptococcus pneumoniae. * Todas las fechas corresponden al año 2017.

curva febril; se realizó punción lumbar de control después de 1 semana afebril, el líquido cefalorraquídeo mostró disminución de proteinorraquia $(100 \mathrm{mg} / \mathrm{dl})$, glucosa normal, celularidad muy escasa con $100 \%$ mononucleres ( 3 células por $\mathrm{mm} 3$ ) y cultivo negativo. A los 28 días de tratamiento antibiótico fue dada de alta. Continuó seguimiento ambulatorio con completa recuperación y alta definitiva a las 2 semanas de haber egresado del hospital (Tabla 1).

\section{DISCUSIÓN}

Streptococcus pneumoniae es el agente etiológico bacteriano de meningoencefalitis más frecuente en la población pediátrica. Según datos aportados por la Organización Mundial de la Salud, 1,6 millones de niños menores de cinco años presentan un episodio de enfermedad neumocócica invasiva, en la que se incluye la meningitis; produciéndose anualmente entre 12000 a 28000 muertes a nivel mundial ${ }^{[4]}$. Además, debido al fenómeno de reemplazo, se ha observado un incremento de episodios de meningitis neumocócica por serotipos no incluidos en la vacuna heptavalente ${ }^{[5]}$. Dato que deberíamos considerar en nuestro caso, pues la paciente recibió el esquema de inmunización completo con esta vacuna (heptavalente).

En el Perú, en el año 2008 se inició la inmunización con vacuna conjugada heptavalente en poblaciones de pobreza y extrema pobreza, ampliándose la cobertura a nivel nacional un año después ${ }^{[6,7]}$. Posteriormente, en el 2012 , se introdujo la vacuna conjugada 10-valente ${ }^{[7]}$, que contiene los principales serotipos causantes de enfermedad estreptocócica en Latinoamérica ${ }^{[8]}$, y a fines del 2015 fue sustituida por la 13-valente debido a su mayor costo-efectividad. En los diferentes países del mundo en donde se incluyó a la vacuna 13-valente dentro del calendario de inmunización, se registró una disminución global de casos de enfermedad neumocócica invasiva en 50 al 60\%; e incluso, para algunas cepas, la incidencia se redujo hasta en $90 \%{ }^{[8]}$. Esto nos sugiere que sería conveniente que pacientes pediátricos inmunizados con vacuna heptavalente, como nuestra paciente, sean inoculados con una dosis adicional con la vacuna 13-valente y así protegerlos frente a cepas emergentes; todo esto fundamentado en que la transición o "catch up" pueden realizarse para completar los esquemas iniciados con heptavalente, o si el esquema está completo, ampliarlo con una sola dosis adicional ${ }^{[9]}$.

Además del estado de inmunización, existen otros factores de riesgo para desarrollar meningitis neumocócica, como el bajo peso al nacer, la asistencia a guardería y los estados de inmunodeficiencia ${ }^{[10]}$, reportándose hasta un $16 \%$ de incidencia de inmunodeficiencia en niños sanos que desarrollan meningitis bacteriana; la gravedad de la paciente, la persistencia de la fiebre y la lenta evolución clínica hicieron que sospecháramos que nuestra paciente tuviera un estado de inmunosupresión, por lo que se decidió estudiar la 
inmunidad humoral (recuento de inmunoglobulinas) y celular (citometría de flujo) no hallándose alteraciones. No obstante, existen reportes de casos de inmunodeficiencia específica para Streptococcus pneumoniae, la cual se manifiesta con una respuesta deficiente a algunos antígenos contenidos en las vacunas; la cual podría evaluarse mediante el dosaje de la concentración plasmática de anticuerpos específicos a cada serotipo de neumococo, donde la concentración plasmática de $0,35 \mathrm{mg} / \mathrm{ml}$ o más para cada serotipo es un predictor de protección ${ }^{[11]}$. Otras inmunodeficiencias encontradas con frecuencia en estos casos son la deficiencia de la fracción C2 y C3 del complemento, asplenia congénita y agammaglobulinemia ligada a $\mathrm{X}^{[11]}$.

También se debe considerar, que los factores asociados a peor pronóstico y mayor letalidad en casos de meningitis por neumococo son: hipoglucorraquia inferior a $20 \mathrm{mg} / \mathrm{dl}$, proteinorraquia mayor de $250 \mathrm{mg} / \mathrm{dl}$, pleocitosis inferior a 200 células al inicio del cuadro, hemoglobina inferior a $7 \mathrm{~g} / \mathrm{dl} \mathrm{y} \mathrm{escala}$ de Glasgow inferior a $10^{[12]}$, encontrándose presentes cuatro de estos en nuestra paciente. Todos estos factores se han observado con mayor frecuencia en infecciones por cepas agresivas como la $19 \mathrm{~A}, 3$ y $6 \mathrm{~F}^{[13]}$.

La principal limitación en nuestro caso es el no haber podido realizar la detección del serotipo específico de neumococo responsable de la infección, lo cual nos permitiría determinar si se trata de cepas emergentes, cepas no incluidas en la vacuna recibida por la paciente o baja concentración de anticuerpos posvacunales. Podríamos deducir que se trata de una cepa muy agresiva por la rapidez de instauración del cuadro y el tiempo prolongado de fiebre a pesar del tratamiento adecuado según cultivos y antibiogramas. Otro aspecto importante a mencionar es la necesidad de revisar los protocolos locales de manejo de meningitis bacteriana para establecer con claridad la cobertura antibiótica en casos especiales (por ejemplo, postraumatismos craneoencefálicos) y la frecuencia de toma de muestras de líquido cefalorraquídeo para control en casos con pobre respuesta al tratamiento.

Por último, cabe resaltar la necesidad e importancia de implementar una vigilancia activa de los cuadros de meningitis neumocócica en nuestro país, con el objetivo de valorar el impacto de la inclusión de la vacuna 13-valente y se evalúe la necesidad de universalizar su uso (tanto para completar los esquemas iniciados por heptavalente como para reforzar los esquemas completos ${ }^{[14,15]}$ ), pues actualmente hay zonas en nuestro país en las que aún se emplea la 10-valente; y los estudios de costo efectividad realizados demuestran que la vacuna 13-valente es más efectiva en reducir la incidencia de enfermedad neumocócica invasiva. Así también, evaluar la asociación de presentación clínica, evolución, resistencia antimicrobiana y serotipos emergentes, con la finalidad de tomar medidas epidemiológicas oportunas y pertinentes.

\section{REFERENCIAS BIBLIOGRÁFICAS}

1. Thigpen MC, Whitney CG, Messonnier NE, Zell ER, Lynfield R, Hadler $\mathrm{JL}$, et al. Emerging Infections Programs Network. Bacterial meningitis in the United States, 1998-2007. N EngI J Med. 2011;364(21):2016-25.

2. Tsai CJ, Griffin MR, Nuorti JP, Grijalva CG. Changing epidemiology of pneumococcal meningitis after the introduction of pneumococcal conjugate vaccine in the United States. Clin Infect Dis. 2008;46(11):1664-72.

3. Prado V. Enfermedades infecciosas emergentes: ¿un problema nuevo? Rev Med Chile. 1996;124:7-10

4. Instituto de Salud Pública de Chile. Vigilancia de Laboratorio de Streptococcus pneumoniae procedente de Enfermedad Invasora en Chile, 2007 - 2015. Boletín Instituto de Salud Pública de Chile. 2015;5(7):3-17.

5. Hsu HE, Shutt KA, Moore MR, Beall BW, Bennett NM, Craig AS, et al. Effect of pneumococcal conjugate vaccine on pneumococcal meningitis. N Engl J Med. 2009;360(3):244-56.

6. Bolaños Díaz R, Mezones-Holguín E, Fiestas Solórzano V, Suárez Moreno V, Sanabria C. Evaluación económica de las vacunas hepta-, deca- y trece-valente para la prevención de neumonía asociada al Streptococcus pneumoniae en niños menores de 5 años en el Perú. Nota Técnica 2011-6. Lima: Instituto Nacional de Salud; 2011.

7. Ministerio de Salud del Perú. Norma Técnica de Salud que establece el Esquema Nacional de Vacunación 2011. NTS N080-MINSA/ DGSP-V.02. Lima: Ministerio de Salud; 2011.

8. Organización Panamericana de la Salud. Informe Regional de SIREVA II, 2011: datos por país y por grupos de edad sobre las características de los aislamientos de Streptococcus pneumoniae, Haemophilus influenzae y Neisseria meningitidis en procesos invasores. Vigilancia sanitaria, prevención y control de enfermedades. Washington, DC: OPS; 2012.

9. Nuorti JP1, Whitney CG; Centers for Disease Control and Prevention (CDC). Prevention of Pneumococcal disease among infants and children - use of 13valent Pneumococcal conjugate vaccine Recommendations of de Advisory Committee on Inmunization Practices (ACIP). MMWR Recomm Rep. 2010;59(RR-11):1-18.

10. Hjuler T, Wohlfahrt J, Simonsen J, Kaltoft MS, Koch A, Kamper-Jorgersen $M$, et al. Perinatal and crowding-related risk factors for invasive pneumococcal disease in infants and young children: a populationbased case-control study. Clin Infect Dis. 2007;44(8):1051-6.

11. Mascini EM, Jansze M, Schellekens J, Musser JM, Faber J, VerhoefVerhage L, et al. Invasive group A streptococcal disease in The Netherlands: evidence for a protective role of anti-exotoxin A antibodies. J Infect Dis. 2000;181(2):631-8.

12. Lovera D, Aranda C, Duarte M, Apodaca S, Acuña J, Arbo A. Predicción de la mortalidad de la meningitis neumocóccica en niños. Pediatr (Asunción). 2011;38(2):111-7.

13. Cleary PP, Kaplan E, Handley J, Wlazlo A, Kim M, Hauser AR, et al. Clonal basis resurgence of serious Streptococcus pyogenes disease in the 1980s. Lancet 1992;339(8792):518-21.

14. Lagos R, Muñoz A, Levine M, Lepetic A, Francois N, Yarzabal J, et al. Safety and immunogenicity of the 10-valent pneumococcal nontypable Haemophilus influenzae protein $D$ conjugate vaccine (PHiD-CV) in Chilean children. Hum Vaccin Immunother. 2014;10(3): 757-66.

15. Ministerio de Salud del Perú. Esquema y Calendario Nacional de Vacunación 2012. Directiva Sanitaria Nº 014-MINSA/DGSP-V.01. Lima: Ministerio de Salud; 2012. 